\title{
Animal Models of Human Pathology 2012
}

\author{
Monica Fedele, ${ }^{1}$ Oreste Gualillo, ${ }^{2}$ and Andrea Vecchione ${ }^{3}$ \\ ${ }^{1}$ Istituto di Endocrinologia ed Oncologia Sperimentale (IEOS)-CNR, 80131 Naples, Italy \\ ${ }^{2}$ SERGAS, Instituto de Investigación Sanitaria de Santiago, Santiago University Clinical Hospital, \\ NEIRID Laboratory 9, 15706 Santiago de Compostela, Spain \\ ${ }^{3}$ Human Cancer Genetics Program, Division of Pathology, Comprehensive Cancer Center, \\ The Ohio State University, Columbus, OH 43216, USA
}

Correspondence should be addressed to Monica Fedele, mfedele@unina.it

Received 29 July 2012; Accepted 29 July 2012

Copyright (C) 2012 Monica Fedele et al. This is an open access article distributed under the Creative Commons Attribution License, which permits unrestricted use, distribution, and reproduction in any medium, provided the original work is properly cited.

In this special issue we gather together 23 articles ( 15 reviews, 7 research articles, and 1 methodology report) with diverse information presently available on animal models of human disease.

The use of animal for studying human diseases, from the pathogenesis to the clinical aspects of diagnosis and therapy, has always been a very useful tool and indeed, has contributed enormously to our understanding of the mechanisms of diseases and to the development of numerous drugs.

The 15 comprehensive reviews critically inform the reader of the latest developments in the animal models of diseases such as Parkinson (Blesa et al.), glaucoma (Bouhenni et al.), dermatophytoses (Shimamura et al.), colitis (Perse and Cerar), HCV infections (Carcamo and Nguyen), Glomerulonephritis (Urushihara et al.), and posterior circulation stroke (Lekic and Ani). Moreover, Underbayev et al. provide a clear and concise review on the applications of induced pluripotent stem cells (iPS) for human disease mouse modeling, focusing on the use of microRNA manipulation in the generation and differentiation of iPS. Goldsmith and Jobin propose the use of zebrafish model to study human disease. Moro and colleagues review the significance of mouse models in the study of human cancer progression with particular emphasis on the possible application of patientderived xenograft in the evaluation of chemoresistance, while Mendes-Braz et al. review the mechanisms of hepatic ischemia-reperfusion injury in animal models, and its translation to the clinical practice. Greco et al. describe the basic principles of high resolution imaging ultrasound equipment and the most important applications in preclinical studies, $\mathrm{Li}$ et al. examine different MRI sequences to explore a murine model of intracerebral hemorrhage, whereas Gargiulo et al. provide a review of the use of PET/CT for examining mouse models of myocardial ischemia and Sergi et al. propose an important role of hepatocellular to cholangiocytic metaplasia (as expressed by switch in keratin expression) in the causation of liver graft dysfunction (including cholestasis) in case of ischemia/reperfusion injury, based on personal observations and evidence from the literature. Di Domenico et al. present an exhaustive analysis of the role played by the vascular endothelial growth factor and some cytokines in periodontal ligament remodeling and bone resorption at the pressure side of orthodontic tooth movement.

The research articles deal with the molecular mechanisms involved in the pathogenesis of a particular disease. Vitale et al. report synaptic differences between wild-type and FDDki mice, essentially in the expression levels of selected proteins, in a model of familial dementia; Venancio et al. demonstrate that sleep deprivation alters rat ventral prostate morphology; Neri et al. analyze the effect of colic vein ligature in rats with loperamide-induced constipation, and Allan et al. evaluate the ability of an antimicrobial peripherally inserted central catheter (PICC) to reduce microbial migration and colonization compared to an uncoated PICC. Dunn and Pinkert provide evidence supporting allotopic expression as a tool for mtDNA disease research with implications in development of DNA-based therapeutics, while Mingchang et al. propose a pilot study to examine the use of T1, T2, and T2* weighted images for evaluating hematoma size and extent of edema in mouse brain at high field. 
In further contributions animal models have been used to implement therapeutic approaches. It is the case of the studies (a research article and a methodology report) by Pereira et al. which develop an animal model to study deep second-degree thermal burning and characterize in vivo hydrogel formulation for burn healing.

In conclusion, as it is shown in the collection of contributions assembled in this special issue, we believe that animal models of human pathologies, because of the tremendous bulk of information that they can supply to scientific community, particularly genetic and alternative models, might lead us into a new era of disease research and drug discovery.

\section{Acknowledgments}

We thank all the authors, the reviewers, and the Hindawi Publishing staff for their strong effort in making possible the realization of this project.

Monica Fedele

Oreste Gualillo

Andrea Vecchione 


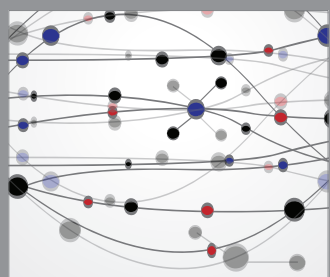

The Scientific World Journal
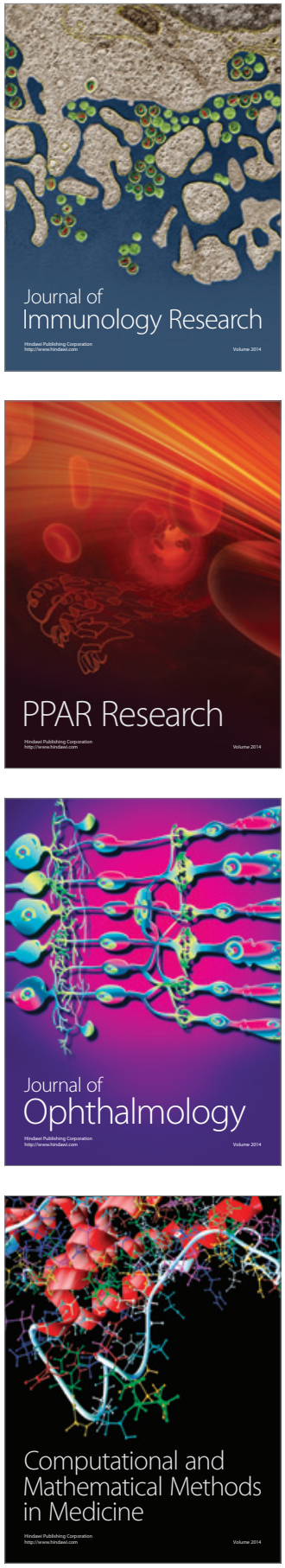

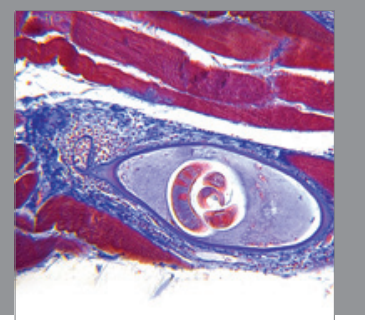

Gastroenterology

Research and Practice
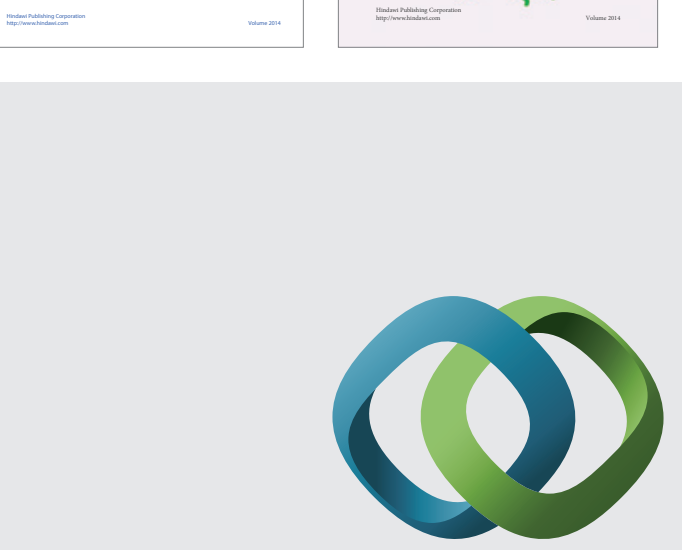

\section{Hindawi}

Submit your manuscripts at

http://www.hindawi.com
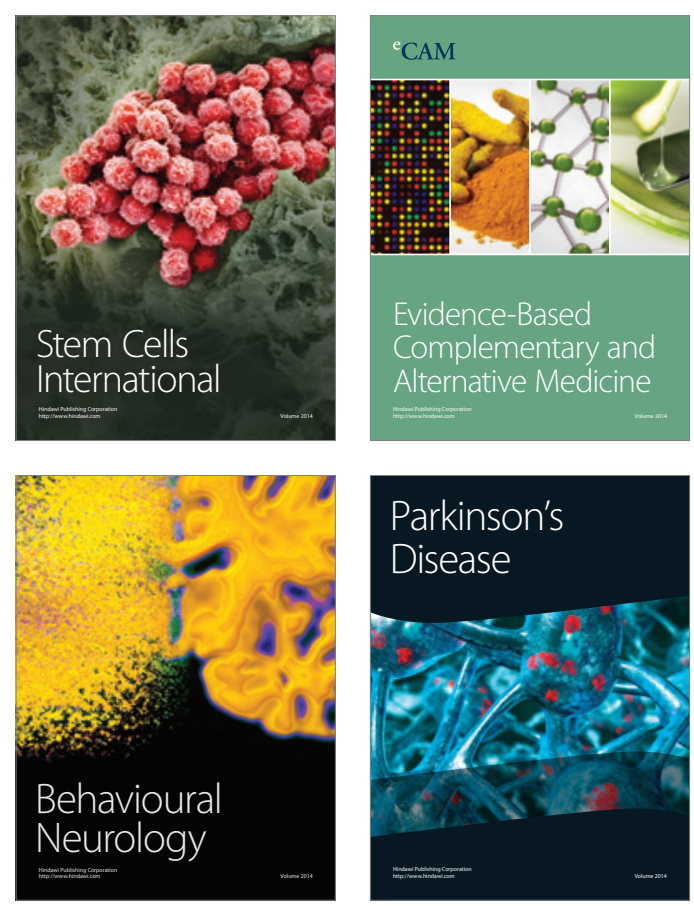

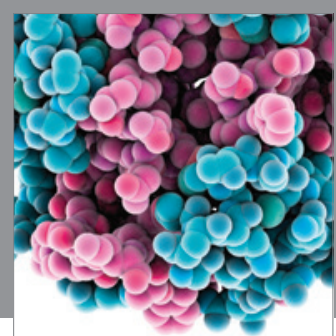

Journal of
Diabetes Research

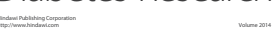

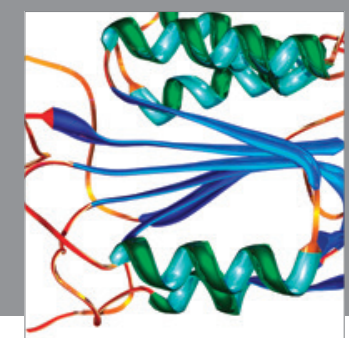

Disease Markers
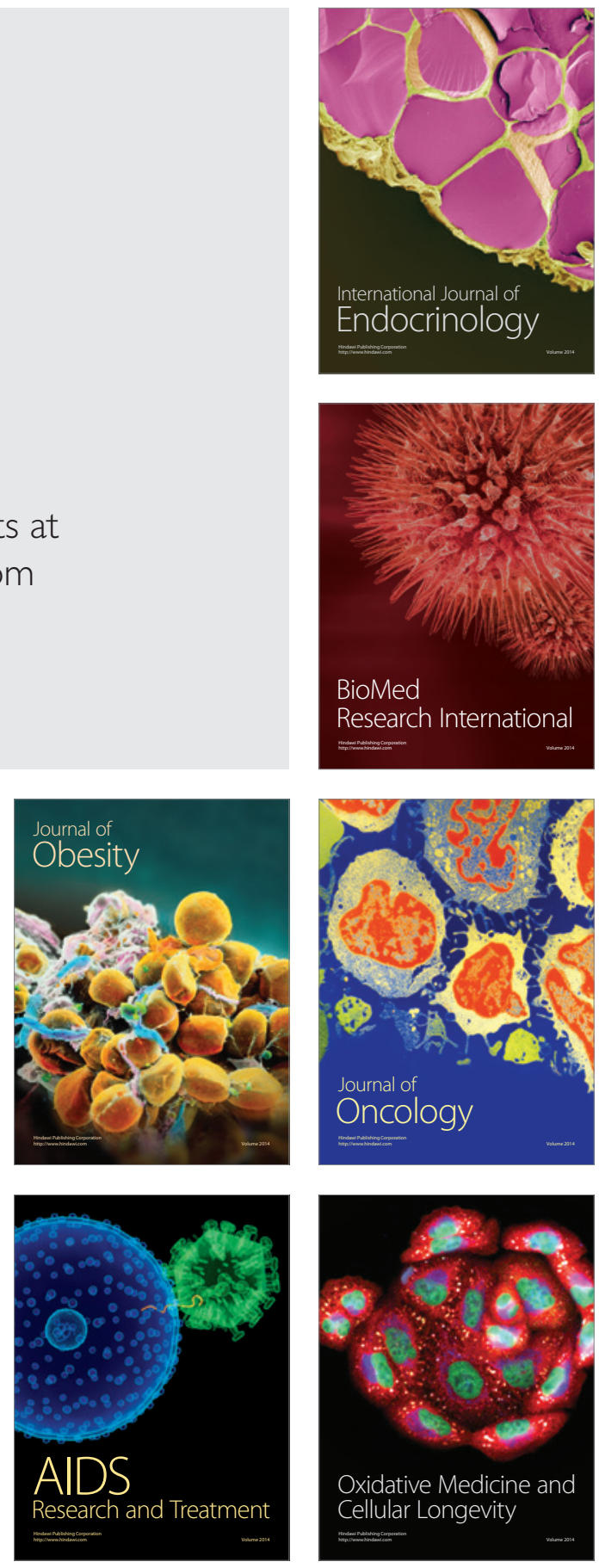